\title{
Data Quality in Biofilm High-Throughput Routine Analysis: Intralaboratory Protocol Adaptation and Experiment Reproducibility
}

\author{
Paula Jorge \\ CEB-Centre of Biological Engineering, LIBRO-Laboratory of Research in Biofilms Rosário Oliveira, University of Minho, \\ Campus de Gualtar, 4710-057 Braga, Portugal \\ Anália Lourenço \\ ESEI-Escuela Superior de Ingeniería Informática, Department of Computer Science, University of Vigo, Ourense, Spain, and \\ CEB-Centre of Biological Engineering, University of Minho, Campus de Gualtar, 4710-057 Braga, Portugal \\ Maria Olívia Pereira \\ CEB-Centre of Biological Engineering, LIBRO-Laboratory of Research in Biofilms Rosário Oliveira, University of Minho, \\ Campus de Gualtar, 4710-057 Braga, Portugal
}

\begin{abstract}
Biofilm research is growing more diverse and dependent on high-throughput technologies, and the large-scale production of results aggravates data substantiation. In particular, experimental protocols are often adapted to meet the needs of a particular laboratory, and no statistical validation of the modified method is provided. This paper discusses the impact of intralaboratory adaptation and non-rigorous documentation of experimental protocols on biofilm data interchange and validation. The case study is a non-standard, but widely used, workflow for Pseudomonas aeruginosa biofilm development considering three analysis assays: the crystal violet (CV) assay for biomass quantification, the 2,3-bis(2-methoxy-4-nitro-5-sulfophenyl)-2 $\mathrm{H}$ tetrazolium-5-carboxanilide inner salt (XTT) assay for respiratory activity assessment, and the colony forming units (CFU) assay for determination of cell viability. The ruggedness of the protocol was assessed by introducing small changes in the biofilm growth conditions, which simulate minor protocol adaptations and non-rigorous protocol documentation. Results show that even minor variations in the biofilm growth conditions may affect the results considerably, and that the biofilm analysis assays lack repeatability. Intralaboratory validation of non-standard protocols is found critical to ensure data quality and enable the comparison of results within and among laboratories.
\end{abstract}

$\mathrm{T}$ The understanding of microbial biofilms is now attracting considerable interest from the clinical community, namely, in studies related to chronic infection, biomaterial-associated infection, and antimicrobial resistance dissemination $(1,2)$. Such research interest is generating a considerable volume of experimental results, but the comparison

Received March 19, 2015. Accepted by AH June 24, 2015

Corresponding author's e-mail: paulajorge@ceb.uminho.pt

DOI: $10.5740 /$ jaoacint.15-066 of results within and across laboratories is still limited (3). Particularly, previous works addressed the need to document biofilm experiments using standard report formats and the creation of standard operating procedures (4-7) to guarantee the repeatability and reproducibility of the experimental protocols $(8-10)$.

Typically, every published study indicates the protocol(s) conducted for the experiment(s) and presents statistical assessments in support of its findings. However, it is a common fact that laboratories often adapt protocols in order to meet the particularities of new environmental scenarios. Protocol adaptation requires rigorous documentation and statistical validation in itself, but such details are usually not provided. This situation has immediate implications in the repeatability and reproducibility of the experiment, and thus in the implementation of interlaboratory comparative studies (11). Consequently, results produced in apparently similar conditions and using the same protocol may vary considerably and lead to quite different conclusions. Moreover, there is evidence that even the most common methods in biofilm analysis may be affected by species-specific issues, e.g., the crystal violet (CV) assay for biomass determination is apparently affected by the matrix composition of Pseudomonas aeruginosa biofilms (12), but it is still widely used in routine biofilm research.

The work presented here aims to explore the impact of protocol adaptation in data repeatability and ruggedness, and to highlight the importance of statistical validation in such scenarios. The case study describes the formation of $P$. aeruginosa biofilms in 96-well microtiter plates and the use of non-standard but conventional methods of biofilm analysis, such as the $\mathrm{CV}$ assay for biomass quantification, the 2,3-bis(2-methoxy-4nitro-5-sulfophenyl)-2H-tetrazolium-5-carboxanilide inner salt (XTT) assay for respiratory activity assessment, and the colony forming units (CFU) assay for cell viability determination. The effect that small variations in common biofilm growth conditions, such as temperature, agitation speed, growth time, and cell subculturing, may have on the variability of $P$. aeruginosa biofilms was tested by assessing the ruggedness of the growth protocol. The repeatability of the three biofilm analysis assays was also evaluated. Results showed that even minor variations in the protocol resulted in significant changes 
Table 1. The three settings for each of the eight operating conditions studied in the ruggedness test ${ }^{a}$

\begin{tabular}{lccc}
\hline & \multicolumn{3}{c}{ Settings } \\
\hline Operating variables & Low & Medium & High \\
\hline Cell subculture & $\mathbf{0}$ & 1 & 2 \\
Time of growth (inoculum), h & 14 & $\mathbf{1 8}$ & 22 \\
Time of growth (biofilm), h & 22 & $\mathbf{2 4}$ & 26 \\
$\begin{array}{l}\text { Initial cell concentration (biofilm), } \\
\text { CFU/mL }\end{array}$ & $4 \times 10^{5}$ & $\mathbf{1 \times 1 0 ^ { 6 }}$ & $2.5 \times 10^{6}$ \\
$\begin{array}{l}\text { Temp. (inoculum and biofilm), }{ }^{\circ} \mathrm{C} \\
\text { Agitation speed } \\
\text { (inoculum and biofilm), rpm }\end{array}$ & 35 & $\mathbf{3 7}$ & 39 \\
$\begin{array}{l}\text { a } \quad \text { The control conditions used in the repeatability assessment are in } \\
\text { bold. }\end{array}$ & & & 140 \\
\end{tabular}

in the parameters of interest and emphasized the critical need to statistically validate non-standardized and modified protocols whenever these are used.

\section{Experimental}

\section{Biofilm Formation}

Overnight cultures of $P$. aeruginosa ATCC 10145 were grown in $20 \mathrm{~mL}$ tryptic soy broth (TSB) (Liofilchem, Roseto degli Abruzzi, Italy) in a $50 \mathrm{~mL}$ shake flask $\left(120 \mathrm{rpm}\right.$, at $37^{\circ} \mathrm{C}$, for 14 h) (N-Biotek Shaker \& Incubator NB-205Q; Gyeonggido, Korea). Subsequently, they were centrifuged $(9000 \times g$, at room temperature, for $5 \mathrm{~min}$ ), resuspended in TSB, and the cell concentration was calculated by a calibration curve relating optical density and CFU concentration (Equation 1):

$$
\mathrm{CFU} / \mathrm{mL}=4 \times 10^{9}-1 \times 10^{8}
$$

where OD is the optical density at $640 \mathrm{~nm}$.

The sample was then diluted until reaching $2 \times 10^{6} \mathrm{CFU} / \mathrm{mL}$. Next, $100 \mu \mathrm{L}$ bacterial suspension were transferred to a flatbottom 96 well polystyrene microtiter plate (Orange Scientific, Braine-l'Alleud, Belgium) to which $100 \mu \mathrm{L}$ TSB/well was added. The plates were incubated aerobically $\left(120 \mathrm{rpm}\right.$, at $37^{\circ} \mathrm{C}$, for $24 \mathrm{~h}$ ) to promote biofilm formation. Afterwards, the liquid content of each well was removed by plate inversion, and the wells were washed twice with $200 \mu \mathrm{L}$ distilled sterile water to remove any planktonic cells. The bacteria that remained attached were analyzed in terms of the adhered biomass, the metabolic activity, and the number of viable cells.

\section{Biomass Evaluation}

After the removal of the planktonic cells, the plates were airdried at room temperature for about $30 \mathrm{~min}$. Biofilm biomass was then quantified by the CV staining assay (13) adapted from Stepanović et al. (14). The biofilms were fixed with $200 \mu \mathrm{L}$ pure methanol (Valente e Ribeiro, Lda., Belas, Portugal)/well for $15 \mathrm{~min}$. Next, the plates were emptied by plate inversion and airdried at room temperature for about $5 \mathrm{~min}$, and the fixed bacteria were stained for 5 min with $200 \mu \mathrm{L} \mathrm{CV}$ [Merck (Darmstadt,

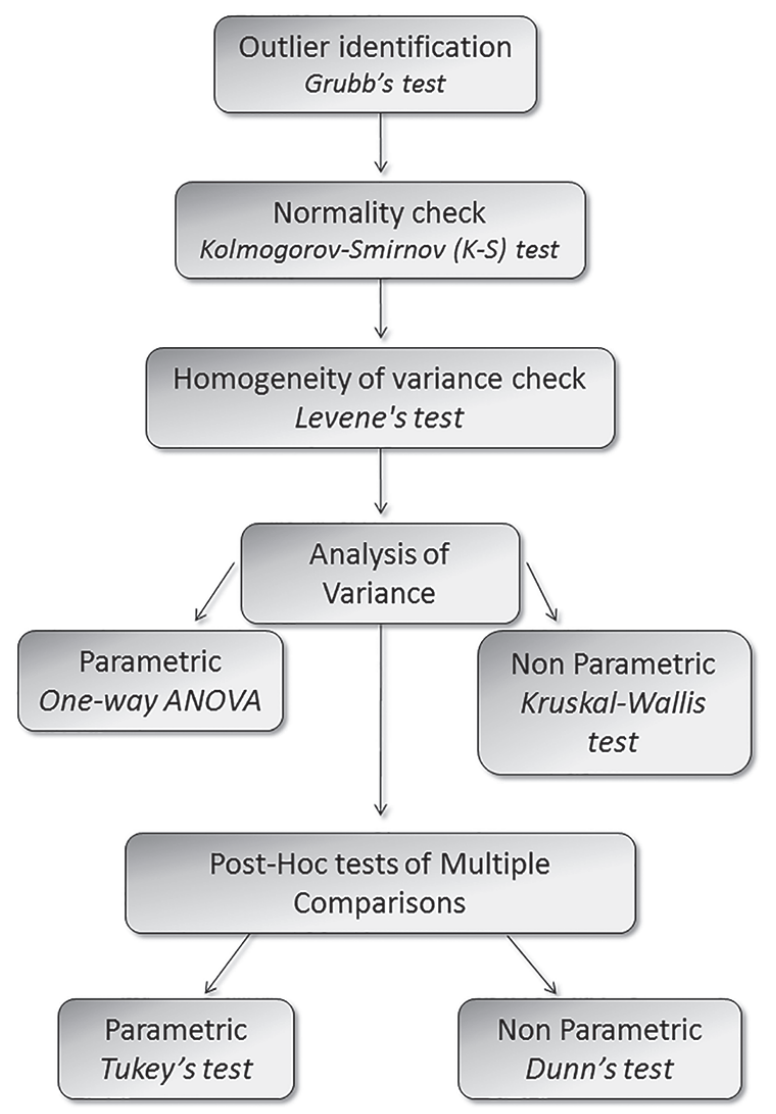

Figure 1. General guideline for the statistical analysis.

Germany) Gram's crystal violet solution, 100\%]/well. Excess stain was carefully rinsed off under low-rate running tap water until the water ran clear. After the plates were air-dried at room temperature for about $10 \mathrm{~min}$., the dye bound to the adherent cells was resuspended with $200 \mu \mathrm{L} \mathrm{33 \%} \mathrm{(v/v)} \mathrm{glacial}$ acetic acid (Fischer Scientific, Porto Salvo, Portugal)/well. The obtained solution was measured for its OD at $570 \mathrm{~nm}$ using a spectrophotometer with microtiter plate reader (Tecan, Model Sunrise-basic Tecan, Grödig, Austria).

\section{Respiratory Activity}

The respiratory activity of the biofilms was measured using the XTT colorimetric assay $(13,15)$ adapted from Stevens and Olsen (16). Biofilms were washed as described in the previous assay, and $200 \mu \mathrm{L}$ combined solution of $150 \mu \mathrm{g} / \mathrm{mL}$ XTT and $10 \mu \mathrm{g} / \mathrm{mL}$ phenazine methosulfate (PMS) was added to each well. Both XTT and PMS were previously diluted separately in distilled sterile water and stored as $1 \mathrm{~mL}$ aliquots at $-20^{\circ} \mathrm{C}$. The combined solution of XTT-PMS was made fresh previous to use and stored at $4{ }^{\circ} \mathrm{C}$ in a light-proof container. After adding the solution, the plates were incubated $\left(120 \mathrm{rpm}\right.$, at $37^{\circ} \mathrm{C}$, 

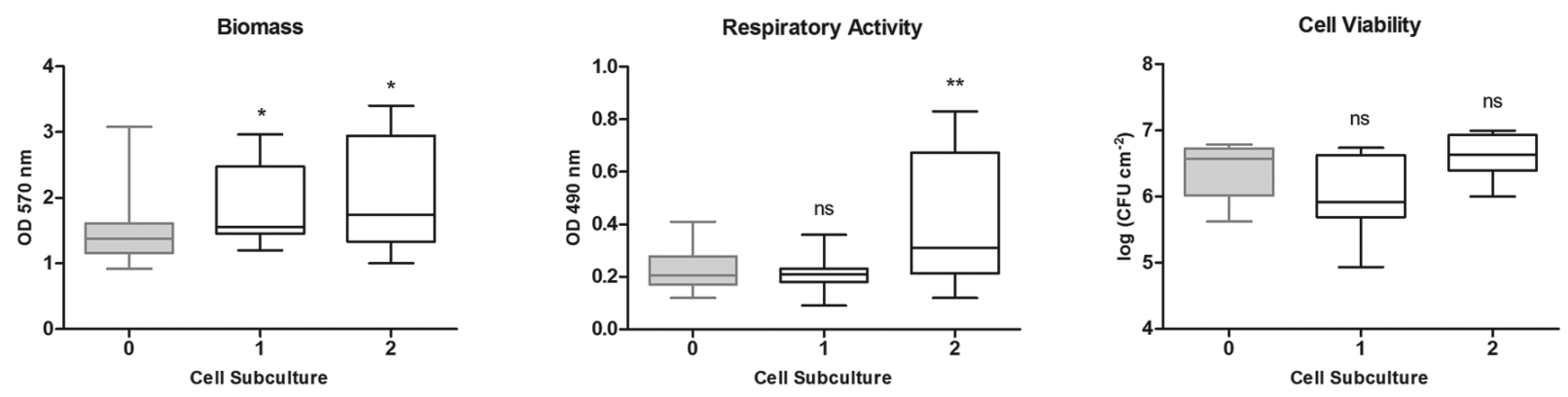

Figure 2. Influence of the cell subculture on the final biofilm. The control condition is in grey and the statistical differences are depicted as: $P<0.001$ (extremely significant ${ }^{* * *}$ ); $0.001<P<0.01$ (very significant ${ }^{* *}$ ); $0.01<P<0.05$ (significant $-{ }^{*}$ ); and $P>0.05$ (not significant - ns). The box-plots show minimum to maximum whiskers, the $25^{\text {th }}$ (bottom of the box), the $50^{\text {th }}$ or median (middle band of the box), and the $75^{\text {th }}$ (top of the box) percentiles.

for $3 \mathrm{~h}$ ) in the dark. The biofilm activity was determined through measurement of OD at $490 \mathrm{~nm}$.

\section{Cell Viability}

Biofilms were detached by sonication for $6 \mathrm{~min}$ (Sonicor Ultrasonic TableTop Cleaner, New York, NY; SC-52, 220 V, $50 / 60 \mathrm{~Hz}, 0.25 \mathrm{~A}$ ) and serially diluted in distilled sterile water to determine the number of CFU (15). After plating the serial dilution on tryptic soy agar, the plates were incubated at $37^{\circ} \mathrm{C}$ overnight prior to enumeration. The number of viable bacterial cells was expressed as $\log \left(\mathrm{CFU} / \mathrm{cm}^{2}\right)$ using Equation 2:

$$
\log _{10}=\left[\left(10^{\mathrm{d}} \times \mathrm{CFU} / 10 \mu \mathrm{L}\right) \times(\mathrm{V} / \mathrm{SA})\right]
$$

where $\mathrm{d}$ is the dilution number (e.g., $0,1,2$, etc. corresponding to the dilution factors of $1,10,100$, etc.), $\mathrm{V}$ is the volume sonicated into $(200 \mu \mathrm{L})$, and SA is the surface area of the microtiter plate's wells $\left(1.53 \mathrm{~cm}^{2}\right)$.

\section{Experimental Design}

In this study, the ruggedness and the repeatability of the biofilm growth and analysis protocol were analyzed. A protocol is considered rugged if the results produced are resistant to minor deviations from the experimental conditions. Here, ruggedness testing evaluated the effect of slight variations in eight common operational conditions considered in the method of biofilm growth, namely, the temperature of microbial growth (inoculum and biofilm), the agitation speed implemented during microbial growth (inoculum and biofilm), the time of growth (inoculum and biofilm), the initial cell concentration, and the cell subculture (Table 1). For the cell subculture condition, three subcultures, corresponding to the initial bacteria retrieved from the preservation cryovial (subculture 0 ) and the following two subcultures of bacteria restreaked from the previous one (subcultures 1 and 2), were considered. Each condition was varied separately in at least three independent experiments with a minimum of four sample replicates each. These changes in the conditions were small and simulated minor adaptations of the protocol or some disregard in terms of their tight control, which is not unusual in laboratorial routine, either by operator or equipment faultiness.

On the other hand, a protocol is considered repeatable if, in similar conditions, the results within the same experiment and between experiments have low variance. In order to determine the repeatability, the group of experiments conducted in the control conditions (Table 1) was analyzed. The control conditions were as stated in the biofilm growth protocol described in the Experimental section. A total of 18 independent experiments with eight sample replicates each were carried out for biomass and respiratory activity assessment, and the
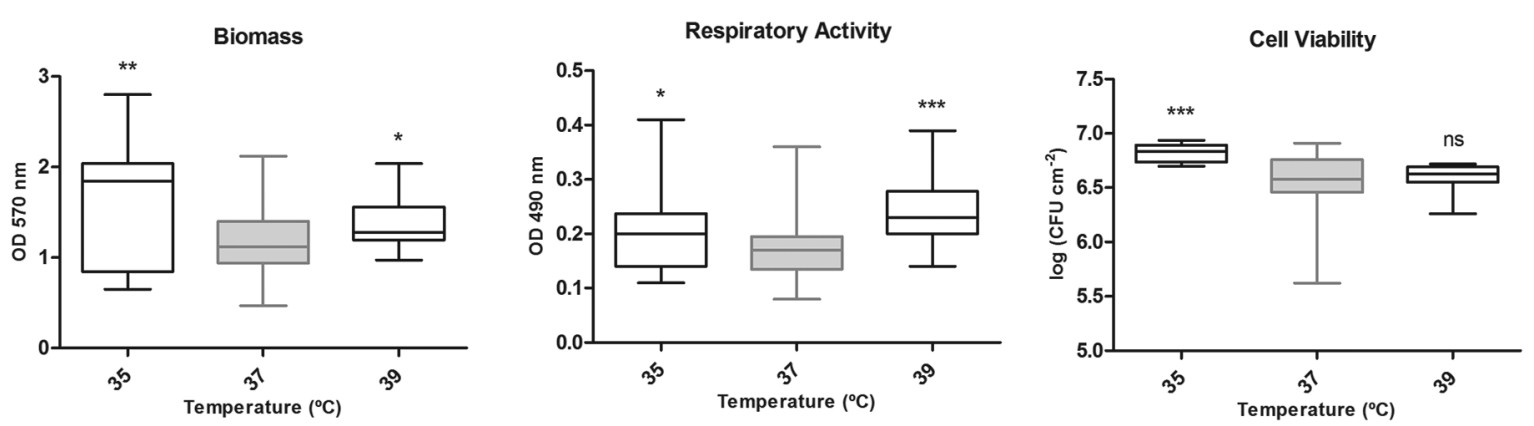

Figure 3. Influence of the temperature of inoculum growth on the final biofilm. The control condition is in grey and the statistical differences are depicted as: $P<0.001$ (extremely significant $-{ }^{* *}$ ); $0.001<P<0.01$ (very significant $-{ }^{* *}$ ); $0.01<P<0.05$ (significant $-{ }^{*}$ ); and $P>0.05$ (not significant $-\mathrm{ns}$ ). The box-plots show minimum to maximum whiskers, the $25^{\text {th }}$ (bottom of the box), the $50^{\text {th }}$ or median (middle band of the box), and the $75^{\text {th }}$ (top of the box) percentiles. 

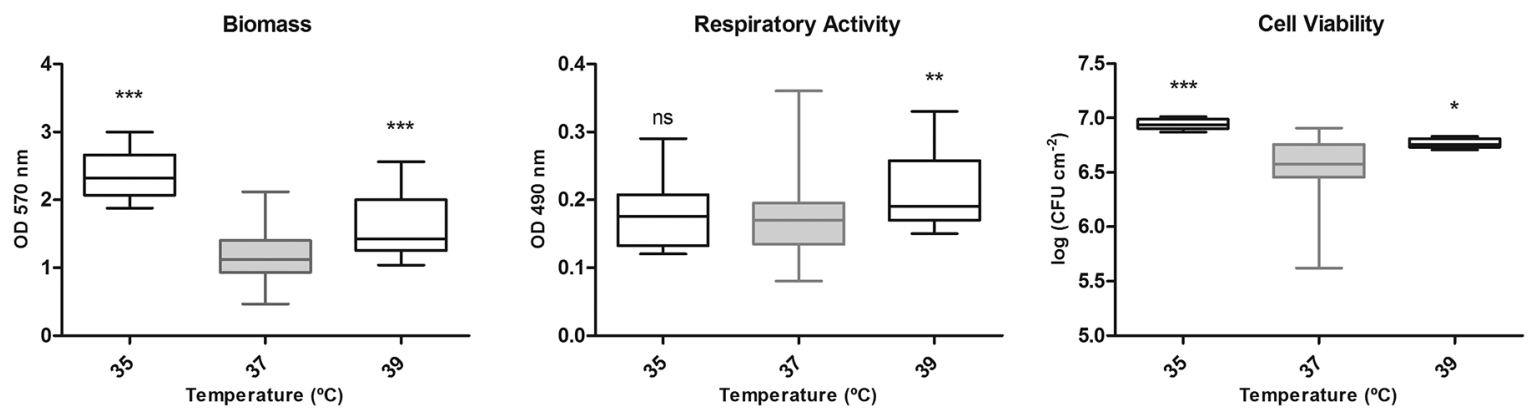

Figure 4. Influence of the temperature of biofilm growth on the final biofilm. The control condition is in grey and the statistical differences are depicted as: $P<0.001$ (extremely significant ${ }^{* * *}$ ); $0.001<P<0.01$ (very significant $-{ }^{* *}$ ); $0.01<P<0.05$ (significant $-{ }^{*}$ ); and $P>0.05$ (not significant - ns). The box-plots show minimum to maximum whiskers, the $25^{\text {th }}$ (bottom of the box), the $50^{\text {th }}$ or median (middle band of the box), and the $75^{\text {th }}$ (top of the box) percentiles.

determination of viable cells encompassed 17 experiments with four sample replicates each.

\section{Statistical Analysis}

The statistical analysis for this study was performed following a simple statistical workflow (Figure 1) as follows: outliers were identified and removed by applying Grubbs' test $(17,18)$, data normality was checked using the Kolmogorov-Smirnov test (19), and data homoscedasticity was checked using Levene's test (20); the analysis of variance (ANOVA) test (21) was applied to the normally distributed data, and the KruskalWallis test (22) was applied otherwise. Multiple comparison analysis relied on Tukey's test (23) and Dunn's test (24) for parametric and non-parametric testing, respectively. The statistical analysis was performed using Microsoft Excel and GraphPad software (25). This work does not address technicianto-technician variability, i.e., all the tests were performed by the same operator.

Assessments for repeatability were based on the results of the one-way ANOVA and the repeatability SD (Sr; Equation 3):

$$
S_{r}=\sqrt{\sigma_{e}^{2}+\sigma^{2} b t w}
$$

ANOVA assesses two variances: between experiment variance $\left(\sigma_{b t w}^{2}\right)$, which shows the differences between experiment means, and within experiment variance $\left(\sigma_{e}^{2}\right)$, which shows the differences among data within the same group. The $\sigma_{\text {btw }}^{2}$ can be explained by a systematic variation due to treatment or by chance due to non-systematic individual differences in data or experimental error. On the other hand, the $\sigma^{2}$ e can only be explained by experimental error. The calculation of $\mathrm{Sr}$ can be interpreted as the difference between a single experiment and the mean across many independent, identical experiments. Small values of $S r$ indicate good repeatability. The percentage of influence of each variance, $\sigma_{\text {btw }}^{2}$ and $\sigma^{2}$, can be further inspected. The $\sigma^{2}$ btw is estimated by Equation 4:

$$
\sigma^{2}{ }_{b t w}=\sigma^{2}+3 \sigma_{t}^{2}
$$

where $\sigma_{t}^{2}$ is the variance due to treatment effect. Total variance $\left(\sigma_{\text {total }}^{2}\right)$ is the sum of $\sigma_{\mathrm{e}}^{2}$ and $\sigma^{2}$. The percentage of $\sigma_{\mathrm{e}}^{2}$ and $\sigma^{2}$, relative to $\sigma_{\text {total }}^{2}$, can be then easily calculated (26).

\section{Results and Discussion}

\section{Response to Variations in the Biofilm Growth Conditions}

Results show statistically significant differences in the output data regarding small alterations of some of the tested conditions. Specifically, cell subculture (Figure 2), temperature of inoculum (Figure 3), temperature of biofilm growth (Figure 4), and the agitation speed of the biofilm (Figure 5) resulted in different
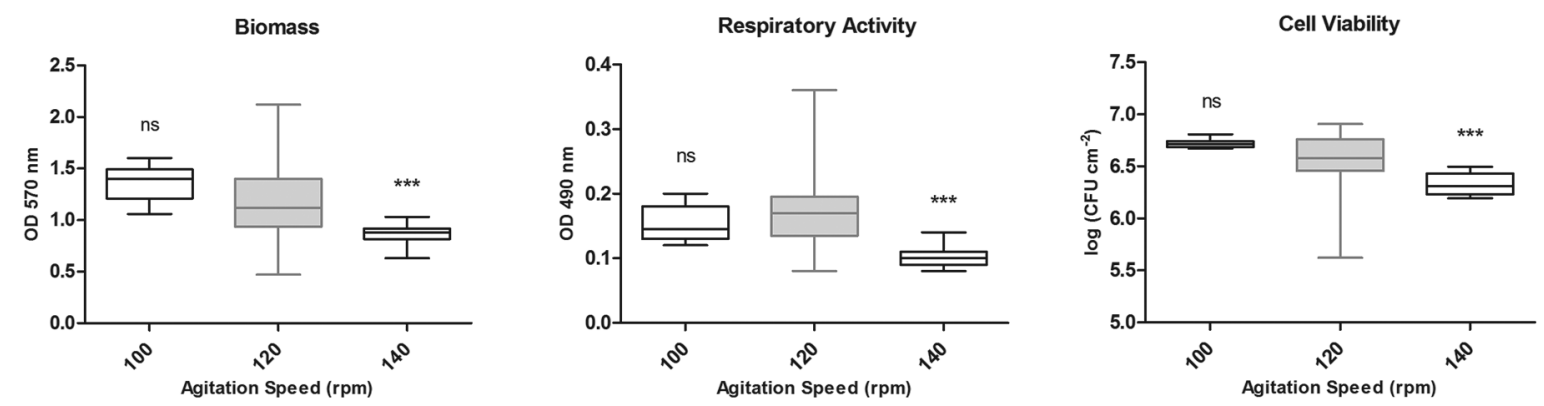

Figure 5. Influence of the agitation speed in the biofilm growth on the final biofilm. The control condition is in grey and the statistical differences are depicted as: $P<0.001$ (extremely significant $-{ }^{* * *}$ ); $0.001<P<0.01$ (very significant ${ }^{* *}$ ); $0.01<P<0.05\left(\right.$ significant $-{ }^{*}$ ); and $P>0.05$ (not significant - ns). The box-plots show minimum to maximum whiskers, the $25^{\text {th }}$ (bottom of the box), the $50^{\text {th }}$ or median (middle band of the box), and the $75^{\text {th }}$ (top of the box) percentiles. 

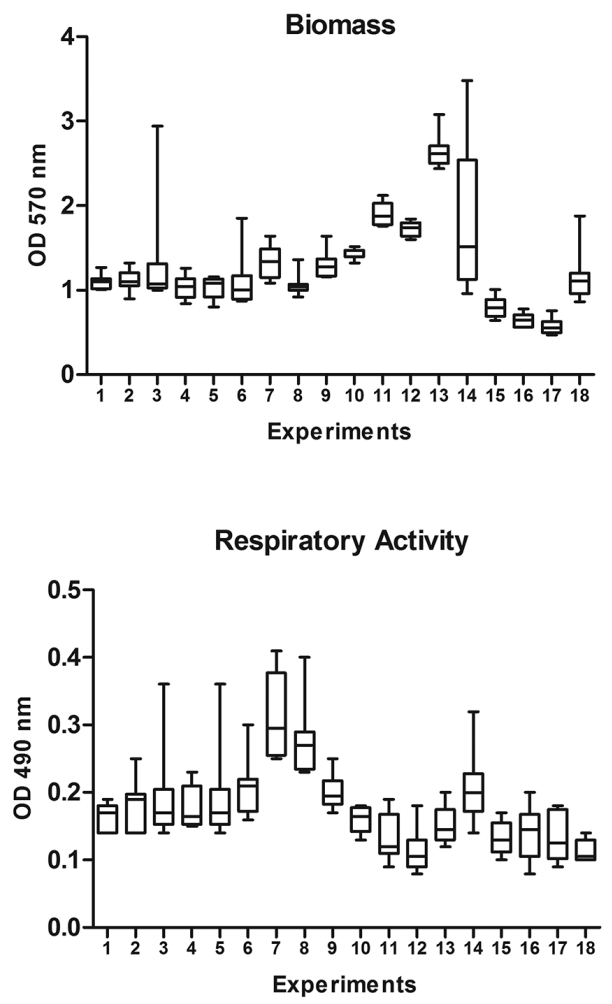

Cell Viability

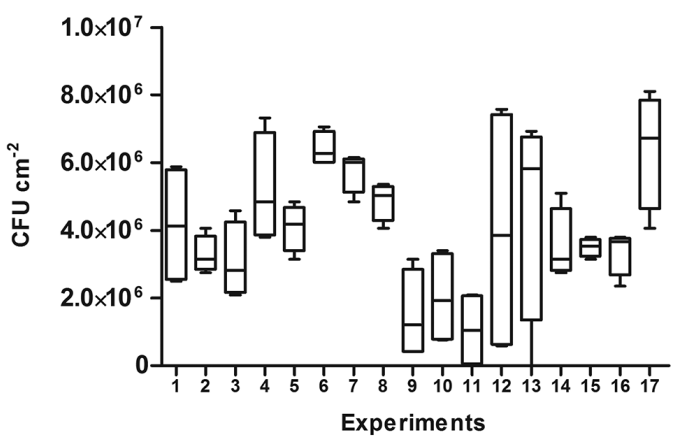

Figure 6. Repeatability data dispersion for the three analysis assays: CV (biomass), XTT (respiratory activity), and CFU (cell viability). The box-plots show minimum to maximum whiskers, the $25^{\text {th }}$ (bottom of the box), the $50^{\text {th }}$ or median (middle band of the box), and the $75^{\text {th }}$ (top of the box) percentiles.

biofilms due to the small changes in the growth conditions. In this study, the ruggedness and the repeatability of the biofilm growth and analysis protocol were analyzed for the second subculture when compared with subculture 0 (Figure 2). Additionally, changes in temperature from $37^{\circ} \mathrm{C}$ to both 35 and $39^{\circ} \mathrm{C}$ caused significant to extremely significant increases in the values of biomass, respiratory activity, and viable cells, both in the inoculum (Figure 3) and in the biofilm (Figure 4). Finally, the increase in agitation speed for the biofilm growth from 120 to $140 \mathrm{rpm}$ decreased extremely significantly the three previous parameters of analysis (Figure 5). Small alterations in the remaining operating conditions, i.e., time of inoculum and biofilm growth, initial cell concentration, and agitation speed for the inoculum growth, did not cause any statistically significant differences in the three parameters of biofilm analysis (data not shown).

In more detail, e.g., the biomass and respiratory activity of the biofilm increased significantly from subculture 0 to subcultures 1 and 2 (Figure 2). This can be related to the fact that stored cells may show different metabolic states and other acquired characteristics throughout the storage time and from subculture to subculture. This means that the type and time of storage can influence the cells in such a way that they can produce different biofilms, even though it is a preliminary step of the biofilm growth protocol and it is often not specified in the protocols.

Also, the biomass, respiratory activity, and cell viability of the biofilm were extremely significantly lower when the agitation speed under which the biofilm was formed was raised from 120 to $140 \mathrm{rpm}$ (Figure 5). This may be due to the impairment of the adhesion of the cell to the surface because of the agitation. The process of adhesion is crucial in order for cells in the planktonic (non-attached) state be able to adhere and form biofilms. In fact, even after cell adhesion, the biofilm formation could have been delayed due to the removal of cells from the upper layers of the biofilm due to the shear stress forces.

These results show that small variations in the conditions of the protocol can cause different results, which highlights the importance of the impact that arises from neglecting the tight control of such growth conditions. This may happen during a protocol adaptation, in which conditions may be altered in order to meet specific needs, or during a protocol's implementation, in which operators may both misinterpret some instructions if they are poorly described and disregard others. Of course the seriousness of such scenarios is lowered when experiments are consistently performed within the same study, by the same operator, and within the same laboratory. If the conclusions are only taken for comparisons within these parameters, the protocol used does not need to conform to those of other studies. However, the comparison of results both intralaboratory and interlaboratory is key in current biofilm research, given the ultimate importance of such studies in today's healthcare investigation, for instance.

\section{Comparing Multiple Measurements}

Data dispersion throughout the experiments performed in the control conditions (Table 1) suggests that the three biofilm analysis assays were affected by some degree of variability (Figure 6). By analyzing the values of $S_{\mathrm{r}}$ (Table 2), it is clear that these values are higher than desirable (they are not far apart from the mean values), and that differences between the experiments greatly influenced the variability of the three methods. For biomass analysis, the value of the $S_{\mathrm{r}}$ is $0.97 \mathrm{OD}$ at $570 \mathrm{~nm}$, which corresponds to a $90.7 \%$ of variability attributed to differences between experiments and $9.32 \%$ to error variability within experiments. For respiratory activity, the value of the $S_{\mathrm{r}}$ is $0.131 \mathrm{OD}$ at $490 \mathrm{~nm}$, corresponding to $75.7 \%$ of variability due to differences between experiments and $24.3 \%$ due to errors within experiments. For cell viability, the value of the $S_{\mathrm{r}}$ is $2.84 \times 10^{6} \mathrm{CFU} / \mathrm{cm}^{2}$, i.e., $66.5 \%$ of variability is due to differences between experiments and $33.5 \%$ to errors within experiments. Furthermore, results show that some methods of analysis may not be adequate to the study of certain organisms. Notably, the CV assay, which is currently widely used for this purpose, showed compromised repeatability for the biomass 
Table 2. Statistical evaluation of experiment repeatability

\begin{tabular}{|c|c|c|c|c|}
\hline & & \multicolumn{3}{|c|}{ Assay } \\
\hline & & Biomass & Respiratory activity & Cell viability \\
\hline \multirow[t]{4}{*}{ Number of } & Experiments & 18 & 18 & 17 \\
\hline & Replicates & 8 & 8 & 4 \\
\hline & Data points & 144 & 144 & 68 \\
\hline & Outliers & 11 & 3 & 2 \\
\hline Normality & & Yes $(P \geq 0.03)$ & Yes $(P \geq 0.05)$ & Yes $(P \geq 0.02)$ \\
\hline Homogeneity of variance & & Yes $(P>0.05)$ & Yes $(P>0.05)$ & Yes $(P>0.001)^{a}$ \\
\hline Mean & & 1.16 & 0.172 & $4.46 \times 10^{6}$ \\
\hline SD & & 0.370 & 0.057 & $1.64 \times 10^{6}$ \\
\hline \multirow[t]{6}{*}{ ANOVA } & $p$ value & $P<0.0001$ & $P<0.0001$ & $P<0.0001$ \\
\hline & $\sigma_{e}^{2}$ & 0.030 & 0.002 & $1.02 \times 10^{12}$ \\
\hline & $\sigma_{b t w}^{2}$ & 0.911 & 0.016 & $7.06 \times 10^{12}$ \\
\hline & $\sigma_{t}^{2}$ & 0.294 & 0.005 & $2.02 \times 10^{12}$ \\
\hline & $\sigma_{\text {total }}^{2}$ & 0.324 & 0.006 & $3.03 \times 10^{12}$ \\
\hline & $S_{r}$ & 0.970 & 0.131 & $2.84 \times 10^{6}$ \\
\hline
\end{tabular}

\footnotetext{
Values are in OD $570 \mathrm{~nm}$ and OD $490 \mathrm{~nm}$ units for the CV and XTT assays, respectively. In the case of homoscedasticity, in the cell viability dataset, homogeneity of variance was not encountered in the unit log $\left(\mathrm{CFU} / \mathrm{cm}^{2}\right)$, so the logs were retrieved and homogeneity of variance was found with the data in CFU $/ \mathrm{cm}^{2}$. Literature states that when Levene's test fails, often the best approach is to transform the data to logarithms or reciprocals, restoring equal variance (26). All values are presented with at least three significant digits.
}

quantification of $P$. aeruginosa biofilms, as already pointed out by some authors (12). Once again, these results reflect the necessity of properly validating the adaptation of protocols, such as these three biofilm analysis assays, to different experimental scenarios. In fact, the inexistence of standard protocols for each of the assays chosen has led to many common variations in the literature resulting from different protocol adaptations. For instance, the analysis of biofilm biomass by the CV assay may be done by fixing the cells before staining, after staining, or not at all. Also, in the viability CFU assay, there are many possibilities of sonication conditions to remove adhered cells, or cells may be detached by scraping.

\section{Conclusions}

Typically, published studies include a statistical validation of their data results. However, the protocols used to generate those data do not usually undergo the same scrutiny. Most research groups rely on in-house protocols that were adapted at one time or another in order to meet specific needs of investigation, but such protocol alterations have not been statistically validated. Also important is that some protocols are poorly described (e.g., the description of growth overnight rather than indication of the exact number of hours), which may lead to different interpretation of the instructions in the protocol.

This work shows the impact of small variations in the experimental conditions on the repeatability and ruggedness of a widely used and non-standard workflow for P. aeruginosa biofilm growth and analysis. Such small variations can be easily caused by protocol adaptations, errors in laboratorial routine analysis, or misinterpretation of the protocol, and they can lead to statistically different results, namely, in terms of the biofilm's biomass. The three analysis assays (CV, XTT, and $\mathrm{CFU}$ ) showed compromised repeatability, mostly due to between experiment variance, which was most evident in the case of the CV staining assay.

The quality of the data acquired using these analysis assays impairs the establishment of intralaboratory and interlaboratory comparisons. It stands to reason that intralaboratory protocol adaptations, common in everyday biofilm research, should be statistically validated and formally described in order to guarantee data quality and ensure protocol reproducibility within and across laboratories.

\section{Acknowledgments}

We thank the Portuguese Foundation for Science and Technology (FCT) the strategic funding of UID/BIO/04469/2013 unit. The authors also acknowledge the support by FCT and the European Community fund FEDER, through Program COMPETE, under the scope of the Projects AntiPep PTDC/SAUSAP/113196/2009 (FCOMP-01-0124-FEDER-016012), RECI/ BBB-EBI/0179/2012 (FCOMP-01-0124-FEDER-027462), the Projects "BioHealth-Biotechnology and Bioengineering approaches to improve health quality," NORTE-07-0124FEDER-000027, co-funded by the Programa Operacional Regional do Norte (ON.2-O Novo Norte), QREN, FEDER, and the Agrupamento INBIOMED from DXPCTSUG-FEDER unha maneira de facer Europa (2012/273). The research leading to these results has received funding from the European Union's Seventh Framework Programme FP7/REGPOT-2012-2013.1 under grant agreement No. 316265, BIOCAPS. This document reflects only the author's views, and the European Union is not liable for any use that may be made of the information contained herein. The authors also acknowledge the Ph.D. Grant of Paula Jorge, Ref. SFRH/BD/88192/2012. 


\section{References}

(1) Høiby, N., Bjarnsholt, T., Givskov, M., Molin, S., \& Ciofu, O. (2010) Int. J. Antimicrob. Agents 35, 322-332. http://dx.doi. org/10.1016/j.ijantimicag.2009.12.011

(2) Jorge, P., Lourenço, A., \& Pereira, M.O. (2012) Biofouling 28 , 1033-1061. http://dx.doi.org/10.1080/08927014.2012.728210

(3) Huang, Y., \& Gottardo, R. (2012) Br. Bioinform. 14, 391-401. http://dx.doi.org/10.1093/bib/bbs078

(4) ASTM (2012) E2562-12 Standard Test Method for Quantification of Pseudomonas aeruginosa Biofilm Grown with High Shear and Continuous Flow Using CDC Biofilm Reactor, ASTM International, West Conshohocken, PA. www.astm.org

(5) ASTM (2012) E2196-12 Standard Test Method for Quantification of Pseudomonas aeruginosa Biofilm Grown with Medium Shear and Continuous Flow Using Rotating Disk Reactor, ASTM International, West Conshohocken, PA. www .astm.org

(6) ASTM (2013) E2647-13 Standard Test Method for Quantification of a Pseudomonas aeruginosa Biofilm Grown Using a Drip Flow Biofilm Reactor with Low Shear and Continuous Flow, ASTM International, West Conshohocken, PA. www.astm.org

(7) ASTM (2012) E2799-12 Standard Test Method for Testing Disinfectant Efficacy Against Pseudomonas aeruginosa Biofilm Using the MBEC Assay, ASTM International, West Conshohocken, PA. www.astm.org

(8) Hamilton, M.A. (2010) KSA-SM-03 Desirable Attributes of a Standardized Method, Standardized Methods: Testing Surface Disinfectants, Center for Biofilm Engineering at Montana State University, Bozeman, MT. http://www.biofilm.montana.edu/ content/ksa-sm-03

(9) Parker, A.E., Walker, D.K., Goeres, D.M., Allan, N., Olson, M.E., \& Omar, A. (2014) J. Microbiol. Methods 102, 55-64. http://dx.doi.org/10.1016/j.mimet.2014.04.013

(10) Buckingham-Meyer, K., Goeres, D.M., \& Hamilton, M.A (2007) J. Microbiol. Methods 70, 236-244. http://dx.doi. org/10.1016/j.mimet.2007.04.010

(11) Annis, D.H., \& Craig, B.A. (2005) Diagn. Microbiol. Infect. Dis. 53, 61-64. http://dx.doi.org/10.1016/j. diagmicrobio.2005.03.012

(12) Peeters, E., Nelis, H.J., \& Coenye, T. (2008) J. Microbiol. Methods 72, 157-165. http://dx.doi.org/10.1016/j. mimet.2007.11.010
(13) Lopes, S.P., Machado, I., \& Pereira, M.O. (2011) J. Ind. Microbiol. Biotechnol. 38, 133-140. http://dx.doi.org/ doi:10.1007/s10295-010-0838-y

(14) Stepanović, S., Vuković, D., Dakić, I., Savić, B., \& ŠvabićVlahović, M. (2000) J. Microbiol. Methods 40, 175-179. http://dx.doi.org/doi:10.1016/S0167-7012(00)00122-6

(15) Machado, I., Lopes, S.P., Sousa, A.M., \& Pereira, M.O. (2012) J. Basic Microbiol. 52, 43-52. http://dx.doi.org/doi:10.1002/ jobm. 201100137

(16) Stevens, M.G., \& Olsen, S.C. (1993) J. Immunol. Methods 157, 225-231. http://dx.doi.org/doi:10.1016/00221759(93)90091-K

(17) Grubbs, F.E. (1969) Technometrics 11, 1-21. http://dx.doi.org/ 10.1080/00401706.1969.10490657

(18) Stefansky, W. (1972) Technometrics 14, 469-479. http://dx.doi .org/10.1080/00401706.1972.10488930

(19) Chakravarti, I.M., Laha, R.G., \& Roy, J. (1967) Handbook of Methods of Applied Statistics. John Wiley \& Sons, New York, NY, pp 392-394

(20) Levene, H. (1960) in Contributions to Probability and Statistics: Essays in Honor of Harold Hotelling, I. Olkin, S.G. Ghurye, W. Hoeffding, W.G. Madow, \& H.B. Mann (Eds), Stanford University Press, Stanford, CA

(21) Snedecor, G.W., \& Cochran, W.G. (1980) Statistical Methods, Iowa State University Press, Ames, IA

(22) Kruskal, W.H., \& Wallis, W.A. (1952) J. Am. Stat. Assoc. 47, 583-621. http://dx.doi.org/10.1080/01621459.1952.10483441

(23) NIST/SEMATECH (2012) e-Handbook of Statistical Methods: Tukey's Method. http://www.itl.nist.gov/div898/handbook/prc/ section4/prc471.htm

(24) Dunn, O.J. (1964) Technometrics 5, 241-252. http://dx.doi.org/ 10.1080/00401706.1964.10490181

(25) GraphPad-Software (2012) What to do When Data Fail Tests for Homogeneity of Variance? http://www.graphpad.com/ support/faqid/1007/

(26) NIST/SEMATECH (2012) e-Handbook of Statistical Methods: What Are Variance Components? http://www.itl.nist.gov/ div898/handbook/prc/section4/prc44.htm 